\title{
Guest Editorial: Understanding of MPN and MDS/MPN based on molecular pathogenesis and clinical aspects
}

\author{
Hironori Harada ${ }^{1}$
}

Received: 20 April 2017 / Accepted: 23 April 2017 / Published online: 26 April 2017

(C) The Japanese Society of Hematology 2017

Myeloproliferative neoplasms (MPN), myelodysplastic syndromes (MDS), and myelodysplastic/myeloproliferative neoplasms (MDS/MPN) are defined as stem cell disorders caused by various gene abnormalities. Recent analysis using next-generation sequencing (NSG) has provided great advances in identifying relationships between gene mutations and clinical phenotypes. Gene mutations affecting RNA splicing machinery, DNA methylation, histone modifications, transcription factors, signal transduction proteins, and components of the cohesion complex participate in the pathogenesis and progression of these myeloid neoplasms [1].

$J A K 2$, calreticulin $(C A L R)$, and $M P L$ mutations which are frequently detected in patients with classical MPN have been shown to constitutively active cytokine receptors. JAK $2^{\mathrm{V} 617 \mathrm{~F}}$ activates erythropoietin receptor, granulocyte colony-stimulating factor receptor, and MPL, whereas the functions of CALR mutants and MPL mutants are MPL activation only. Recently, the molecular mechanisms by which CALR mutant promotes the development of MPN were clarified [2]. Therefore, the development of novel therapeutic targets for MPN with CALR mutant is expected.

MDS/MPN is characterized by overlapped features of bone-marrow failure in MDS and myeloproliferation in MPN. Although much was unknown to the molecular abnormalities of MDS/MPN, gene analysis using NSG is beginning to uncover the gene mutations defining clinical characteristics. According to 2016 World Health

Hironori Harada

hharada@toyaku.ac.jp

1 Tokyo University of Pharmacy and Life Sciences, Hachioji, Tokyo, Japan
Organization (WHO) classification, MDS/MPN includes chronic myelomonocytic leukemia (CMML), atypical chronic myeloid leukemia (aCML), juvenile myelomonocytic leukemia (JMML), MDS/MPN with ring sideroblasts and thrombocytosis (MDS/MPN-RS-T), and MDS/MPNunclassifiable (MDS/MPN-U).

Clinical features of CMML are quite heterogeneous. More than $90 \%$ of CMML patients have one or more gene mutations; however, no disease-specific gene mutation has been identified. Mutations in TET2, SRSF2, ASXL1, and $R A S$ genes are frequently detected and ASXL1 mutations negatively affect the disease outcome. Moreover, it has been clarified that the clonal architecture of CMML is characterized by linear accumulation of mutations [3]. Recently, international consortium perspectives in diagnostic recommendations and response criteria were reported, and clinical reports on CMML, including a new diagnostic method, molecularly integrated CMML-specific prognostic models, and therapeutic trials, are increasing.

A rare subtype of MDS/MPN, aCML, resembles classical CML, but lacking both $B C R-A B L 1$ rearrangement and MPN-associated mutations. Therefore, the treatment by tyrosine kinase inhibitors used for CML is ineffective in aCML. Recently, aCML is better characterized by the molecular abnormalities. SETBP1 which protects SET from protease cleavage is most frequently mutated in aCML. The SETBP1 mutants repress phosphatase 2A activity and promote cellular proliferation, suggesting that the SETBPI mutations activate leukemogenic SETBP1 function in the development of aCML [4].

Ring sideroblasts (RS) are erythroid precursors with abnormal perinuclear mitochondrial iron accumulation. MDS/MPN-RS-T is diagnosed by the presence of RS ( $\geq 15 \%$ of erythroid precursors) and persistent thrombocytosis with platelet count of at least $450 \times 10^{9} / \mathrm{L}$. Mutations 
of the spliceosome gene $S F 3 B 1$ which is associated with the presence of RS are frequently found in patients with MDS/MPN-RS-T. Half of MDS/MPN-RS-T patients have both SF3B1 and JAK2 mutations, suggesting that MDS/ MPN-RS-T has clinical features and molecular abnormalities of both MDS and MPN [5]. Therefore, the development of new molecular target therapies to spliceosome is expected for MDS/MPN patients with spliceosome gene mutations.

This review series in the Progress in Hematology highlight MPN and MDS/MPN based on molecular pathogenesis and clinical aspects. I hope that the reviews by four expert authors will be of great interest to the readers of the International of Hematology.

\section{References}

1. Harada H, Harada Y. Recent advances in myelodysplastic syndromes: molecular pathogenesis and its implications for targeted therapies. Cancer Sci. 2015;106:329-36.
2. Araki M, Yang Y, Masubuchi N, Hironaka Y, Takei H, Morishita $\mathrm{S}$, et al. Activation of the thrombopoietin receptor by mutant calreticulin in CALR-mutant myeloproliferative neoplasms. Blood. 2016;127:1307-16.

3. Itzykson R, Kosmider O, Renneville A, Morabito M, Preudhomme $\mathrm{C}$, Berthon $\mathrm{C}$, et al. Clonal architecture of chronic myelomonocytic leukemias. Blood. 2013;121:2186-98.

4. Makishima H, Yoshida K, Nguyen N, Przychodzen B, Sanada M, Okuno Y, et al. Somatic SETBP1 mutations in myeloid malignancies. Nat Genet. 2013;45:942-6.

5. Lee SC, Dvinge H, Kim E, Cho H, Micol JB, Chung YR, et al. Modulation of splicing catalysis for therapeutic targeting of leukemia with mutations in genes encoding spliceosomal proteins. Nat Med. 2016;22:672-8. 